\title{
Young Children with Type 1 Diabetes: Sleep, Health-Related Quality of Life, and Continuous Glucose Monitor Use
}

\author{
Manuela Sinisterra, BA, Samantha Hamburger, BS, Carrie Tully, PhD, ${ }^{1,2}$ Emily Hamburger, BA, ${ }^{3}$ \\ Sarah Jaser, $\mathrm{PhD}^{3}$ and Randi Streisand, $\mathrm{PhD}^{1,2}$
}

\begin{abstract}
While children with type 1 diabetes (T1D) and their parents report significant sleep problems, few studies have focused on young children and included health-related quality of life (HRQOL) as an outcome of sleep disturbance. In addition, relatively little is known about the use of diabetes devices, such as continuous glucose monitors (CGMs), in young children and their link with sleep disturbances. This brief report examines the relationship between sleep quality and HRQOL and explores sleep disturbances related to CGM use in a sample of young children with T1D. Data are from the baseline of a behavioral intervention pilot for 46 parents of children ages 2-5 years with T1D. Parents reported on their child's sleep disturbances as a result of nighttime blood glucose monitoring (NBGM). Sleep was measured objectively in a subset of children $(N=11)$ who wore accelerometers for a 5-day period. All parents completed measures of pediatric and parental HRQOL. Greater child sleep disturbance due to NBGM was associated with lower pediatric HRQOL. Child sleep disturbances were negatively associated with parental life satisfaction. In addition, children who used CGM experienced fewer sleep disturbances than those who did not. However, parents of children who used CGM experienced greater sleep disturbances related to a higher frequency of NBGM. Pediatric and parental HRQOL were most related to child sleep disturbances by NBGM. CGM use may be associated with better child sleep, as parents are less likely to wake their child for NBGM, although CGM use may also be associated with greater sleep difficulties in parents. Future studies should further explore the relationship between sleep and technology use and impact on clinical outcomes in young children with T1D and their parents.
\end{abstract}

Keywords: Sleep, Health-related quality of life, Continuous glucose monitoring, Pediatrics.

\section{Introduction}

$\mathbf{T}$ HERE ARE MANY barriers to diabetes management, which can result in poor long-term health outcomes and decreased health-related quality of life (HRQOL). ${ }^{1}$ One previously studied barrier to diabetes management is sleep disturbances. Poor sleep quality impacts diabetes management abilities and general well-being. ${ }^{2,3}$ Children with type 1 diabetes (T1D) have been found to have shorter sleep durations, on average, and higher frequency of sleep disturbances due, in part, to nighttime hyperglycemic or hypoglycemic episodes. ${ }^{4,5}$ In school-aged children, poor sleep quality has been linked to higher HbA1c and greater likelihood of experiencing severe hypoglycemia or diabetic ketoacidosis. ${ }^{3}$ Despite this, few studies have explored sleep in preschool age children with T1D. One study of 3-5 years olds found that children slept for an average of $8 \mathrm{~h}$, which is significantly lower than the $10-13 \mathrm{~h}$ recommended by the National Sleep Foundation for this age. ${ }^{6,7}$ However, no studies to date have examined connections between sleep problems and HRQOL of young children with T1D.

More is known about sleep quality of parents of young children with T1D, which may be a reflection of the burden of care on parents during this developmental period. National

\footnotetext{
${ }^{1}$ Children's National Hospital, Center for Translational Research, Washington, District of Columbia.

${ }^{2}$ The George Washington University School of Medicine, Washington, District of Columbia.

${ }^{3}$ Department of Pediatrics, Vanderbilt University Medical Center, Nashville, Tennessee.
} 
guidelines recommend adults (i.e., parents) get 7-9 h of sleep for optimal health, but parents of children with T1D have reported much shorter sleep duration $(M=6.2 \mathrm{~h} ; M=5.8 \mathrm{~h}){ }^{7,8}$ A common factor affecting sleep and HRQOL among parents of young children with T1D is nighttime blood glucose monitoring (NBGM). Most parents report that they regularly check their child's blood glucose (BG) levels overnight. ${ }^{7-9}$ Related consequences of sleep disturbance from NBGM include mood problems and decreased parental HRQOL. ${ }^{2,3,10}$ The relationship between parental sleep disturbance and NBGM is important to understand to improve HRQOL as well as diabetes management behaviors.

Continuous glucose monitors (CGMs) have been found to positively relate to parent psychosocial functioning, specifically related to family functioning, stress, anxiety, and HRQOL. ${ }^{11}$ However, relatively little is known about the impact of CGM on child and parent sleep. Although being relieved of NBGM duties might improve sleep quality, CGM use has been found to negatively impact parent sleep due to device alarms and continued worry about hypoglycemia. ${ }^{12-14}$ Studies have yet to present evidence on the impact of CGM use on child sleep disturbance. We aim to address a gap in the literature by exploring ways, in which CGM use, in general, might relate to sleep disturbances and HRQOL in very young children with T1D.

The current study describes sleep characteristics of young children with T1D and explores how child sleep and NBGM disturbances relate to pediatric and parental HRQOL, while considering the relationship with CGM use.

\section{Methods}

\section{Participants}

The current study was reviewed and approved by the Institutional Review Board of a pediatric hospital in a metropolitan area. Forty-six parent-child dyads (88.9\% mothers, $66.7 \%$ Caucasian, $36.1 \%$ female child) were recruited to participate in a larger behavioral intervention on nutrition and physical activity in young children with T1D. Children were eligible if they were aged 2-5 and had been diagnosed with T1D for $>1$ year. Out of 89 eligible families, 46 enrolled $(51.7 \%)$ and the sample was representative of the clinic population. Only baseline data are presented in this article. Participants were asked to complete a series of psychosocial questionnaires. Blood glucose values were collected via downloaded BG monitors or CGMs. Differences of CGM use across demographic characteristics can be found in Table 1. As a secondary analysis within the study, in the second half of the recruitment period, 13 families were asked if they would be interested in wearing their accelerometer overnight to track sleep patterns. Eleven of the 13 parent-child dyads (84.6\%; 90.9\% mothers, $81.8 \%$ Caucasian, $45.5 \%$ female child, $100 \%$ CGM use) agreed and children wore accelerometers overnight for a minimum of four nights.

\section{Measures}

Sleep. Parents were asked about their child's sleep schedule and their NBGM routine. They reported their child's average sleep and wake times and were asked about sleep disturbances related to NBGM, with specific questions listed below.
Table 1. Demographic Characteristics, Health-Related Quality of Life, and Differences in Continuous Glucose Monitor Use

\begin{tabular}{|c|c|c|c|c|}
\hline & \multicolumn{2}{|c|}{$\begin{array}{l}\text { Accelerometry } \\
\quad(\mathrm{N}=11)\end{array}$} & \multicolumn{2}{|c|}{$\begin{array}{l}\text { Full sample } \\
(\mathrm{N}=46)\end{array}$} \\
\hline & M & $S D$ & M & $S D$ \\
\hline Parent age (years) & 39 & 4.5 & 36.5 & 6.2 \\
\hline Child age (years) & 4.7 & 0.5 & 4.7 & 0.9 \\
\hline Hemoglobin A1c (\%) & 7.8 & 0.8 & 8.3 & 1.0 \\
\hline Diagnosis duration (years) & 2.2 & 0.7 & $2.0^{\mathrm{a}}$ & 0.9 \\
\hline PDQOLQ & 33.6 & 6.3 & 33.4 & 8.9 \\
\hline $\begin{array}{l}\text { PedsQL } \\
\text { Diabetes problems }\end{array}$ & 61 & 15.5 & 60.5 & 13.7 \\
\hline $\begin{array}{l}\text { PedsQL } \\
\text { Treatment I (adherence) }\end{array}$ & 72.2 & 12 & 77.4 & 15.4 \\
\hline $\begin{array}{l}\text { PedsQL } \\
\text { Treatment II (barriers) }\end{array}$ & 71.4 & 11.4 & 79.4 & 13.3 \\
\hline $\begin{array}{l}\text { PedsQL } \\
\text { Worry }\end{array}$ & 46.2 & 28.2 & 45.5 & 26.5 \\
\hline $\begin{array}{l}\text { PedsQL } \\
\quad \text { Communication }\end{array}$ & 72 & 22.4 & 80.9 & 23.5 \\
\hline & $\mathrm{n}$ & $\%$ & $\mathrm{n}$ & $\%$ \\
\hline $\begin{array}{l}\text { Primary caregiver sex } \\
\text { Female }\end{array}$ & 10 & 90.9 & 41 & 89.1 \\
\hline $\begin{array}{l}\text { Child Sex } \\
\text { Female }\end{array}$ & 5 & 45.5 & 17 & 36.9 \\
\hline $\begin{array}{l}\text { Child race/ethnicity } \\
\text { White, non-hispanic }\end{array}$ & 9 & 81.8 & $31^{\mathrm{b}}$ & 67.3 \\
\hline $\begin{array}{l}\text { Marital status } \\
\text { Married }\end{array}$ & 9 & 81.8 & $32^{\mathrm{c}}$ & 69.6 \\
\hline $\begin{array}{l}\text { Employment status } \\
\text { Full-time }\end{array}$ & 8 & 72.7 & 24 & 52.2 \\
\hline $\begin{array}{l}\text { Insulin regimen } \\
\text { Pump }\end{array}$ & 3 & 27.3 & $12^{\mathrm{d}}$ & 26.1 \\
\hline $\begin{array}{l}\text { CGM use } \\
\text { Yes }\end{array}$ & 11 & 100 & 29 & 63.0 \\
\hline
\end{tabular}

${ }^{\mathrm{a}}$ More likely to use CGM if longer time since diagnosis $(P=0.02)$.

${ }^{\mathrm{b}}$ More likely to use CGM if white, non-Hispanic $(P<0.01)$.

${ }^{\mathrm{c}}$ More likely to use CGM if parent is married $(P<0.01)$.

${ }^{\mathrm{d}}$ More likely to use CGM if not on pump, but no children used a pump without CGM $(P<0.01)$.

CGM, continuous glucose monitor; PDQOLQ, parents' diabetes quality-of-life questionnaire; PedsQL, pediatric quality of life, diabetes module; SD, standard deviation.

- After your child goes to sleep, how often do you check his/her blood glucose? (Never, Only if low at bedtime, A few times a month, A few times a week, Most nights, Every night)

- Do you think your child's sleep is disrupted by blood glucose checks? (No, Yes)

- Do you think your own sleep is disrupted by checking your child's blood glucose levels? (No, Yes)

- My child seems tired during the day. (Always, Usually, Sometimes, Rarely, Never)

In addition, ActiGraph wGT3X-BT accelerometry devices were used to objectively measure child sleep for a subset of 
participants. All participants wore the devices for a minimum of four nights, which fulfilled the usability requirements for data analyses. Accelerometer data were scored using the Sadeh algorithm, which uses intervals of rest and activity to assess sleep in youth. ${ }^{15,16}$

Quality of life. The Pediatric Quality of Life, Diabetes Module (PedsQL), is a 28-item measure to understand parents' thoughts about their child's HRQOL. ${ }^{17}$ Items are scored on a 5-point Likert scale and are used to calculate scores for five subscales: Diabetes Problems, Treatment I (Adherence), Treatment II (Barriers), Worry, and Communication. This subscale has demonstrated acceptable internal consistency $(a=0.63-0.81){ }^{17}$ Higher scores on this measure indicate better HRQOL.

The Parents Diabetes Quality-of-Life Questionnaire is a 42-item self-report measure that assesses caregivers' own life satisfaction as impacted by their child's diabetes, the effect of their child's illness on the parent's daily life, and illness-related worries. ${ }^{18}$ The current study used the 16-item Satisfaction subscale. This subscale has been validated and has acceptable internal consistency $(a=0.64-0.89) .{ }^{18}$ Parents were asked to rate how satisfied they feel about their life, within the context of diabetes management, on a 5-point Likert scale. Higher scores indicate greater life dissatisfaction.

Demographic and medical information. Parents reported demographic information for themselves and their child. Study staff reviewed clinic visit records to gather additional medical information. BG data were recorded through downloaded BG meters or CGMs over the course of 5 days.

\section{Results}

For the full sample $(N=46)$, parents reported their child's total sleep time to be an average of $10.4 \mathrm{~h}(\mathrm{SD}=45.6 \mathrm{~min})$, with bed times ranging from 7:00 PM to 11:00 PM and wake times ranging from 6:00 AM to 10:00 AM. Within the accelerometry subset $(N=11)$, parents also reported their child's sleep duration as an average of $10.4 \mathrm{~h}(\mathrm{SD}=41.1$ min). Total sleep time as measured by accelerometry indicated that children slept for an average of $9.8 \mathrm{~h}(\mathrm{SD}=50.1$ min), about a half hour less than what parents of these children reported. The average bed time, as captured by accelerometer, was 9:10 PM (range 7:21-10:52 PM). The average wake time was 7:25 AM (range 6:09-8:23 AM).

Within the total sample $(N=46), 63 \%$ of parents reported checking their child's BG levels at least a few nights per week (responses ranging from "never" $[N=2]$ to "every night" $[N=14]$ ). Differences in frequency of NBGM were found between CGM users and non-CGM users, such that parents of children on CGM reported a higher frequency of NBGM, $t(43)=6.39, P<0.05$. Seventeen percent of parents reported that their child's sleep was disrupted by NBGM, while $78.3 \%$ reported that their own sleep was disrupted by NBGM. Fiftysix percent of parents $(N=26)$ felt that their child typically experienced at least some daytime tiredness (responses ranging from "always" $[N=1]$ to "never" $[N=5])$.

After controlling for demographic characteristics, child sleep disturbance was related to lower pediatric HRQOL, specifically in terms of greater difficulties with diabetesrelated worries $(r=-0.43, P=0.01)$. In addition, when par- ents perceived their child as being more tired during the day, they also perceived their child as having lower HRQOL in terms of diabetes symptoms $(r=0.70, P<0.01)$ and their child's ability to communicate about diabetes management $(r=0.52, P<0.05)$.

An independent samples $t$-test was conducted to compare parent and child sleep quality and HRQOL in CGM users and CGM nonusers. There were significant differences in both parent and child sleep disturbances from NBGM between those who were using CGM and those who were not. Specifically, children who used CGM experienced fewer sleep disturbances than non-CGM users, per parent report $t(44)=3.53, P<0.01$. An inverse relationship was found in parent sleep disturbances from NBGM, indicating that parents of children on CGM experienced greater sleep disturbances, $t(43)=44.24, \quad P<0.01$. However, the results indicated that there were no differences in parent (means for the subscales $=34.3 \pm 8.3$ vs. $M=32.8 \pm 9.4, P>0.1$ ) or child HRQOL (means for the subscales ranging from $58.8 \pm 13.7$ to $77.9 \pm 21.3$ vs. $M$ ranging from $36.9 \pm 25.3$ to $82.6 \pm 11.4$, $P>0.1$ ) between CGM-users versus nonusers.

There were no associations between objective measures of sleep and NBGM or HRQOL within the subset of 11 participants that wore accelerometers. There were large correlations between child daytime tiredness and child HRQOL as related to treatment adherence $(r=0.97, P=0.03)$. There was also a significant relationship between child sleep disturbance from NBGM and greater difficulties related to the child's diabetes symptoms $(r=-0.96, P=0.04)$ and treatment adherence $(r=-0.99, P=0.01)$.

\section{Discussion}

According to both subjective and objective measures of sleep, children in this sample had average sleep times within, yet, on the lower end, of the 10-13 h recommended for this age range. ${ }^{6}$ Past studies have shown much shorter sleep duration in children with $\mathrm{T} 1 \mathrm{D}$, but our findings do not support a notable sleep deficit in this group. In addition, child sleep duration alone did not seem to impact quality of life in children or parents as related to diabetes management. In fact, HRQOL in children and parents was impacted only when children were tired during the day and their parents perceived that their child's sleep was disrupted by NBGM the night before. Although some of the effect sizes of the findings were of moderate size, the results indicate a noteworthy relationship between child sleep and HRQOL. The results also suggest that CGM technology may be helpful for improving child sleep. It is possible that CGM use may assist child sleep duration by minimizing their wake periods throughout the night, given that parents are less likely to wake their child up for NBGM.

Although children in our sample seem to be getting adequate sleep, parent sleep duration, which was not directly measured in this study, might be more negatively influenced by diabetes management and could fall below the recommended $7-9 \mathrm{~h}$ of sleep duration. It would be an unsurprising finding given that diabetes management responsibility in this age group predominantly falls on the parent. The results in the current study indicate that parents of children using CGM are also more likely to engage in more frequent NBGM, which may contribute to greater sleep disturbances. This, along with 
the finding that CGM use in general was related to greater parent sleep disturbances, presents an interesting point to consider when families are deciding whether or not to start diabetes device use.

These results suggest that parents of young children may be most in need of sleep adjustments rather than the children themselves. The findings are promising examples of the ways in which technology use may serve as a tool for improving sleep and subsequent HRQOL in children with T1D, while highlighting the potential negative implications it may have on parent sleep and HRQOL.

\section{Limitations and future directions}

To our knowledge, this was one of the first studies to use both subjective and objective measures of sleep in very young children with T1D. However, future research should include a validated parent-report sleep measure, larger sample of children wearing accelerometers overnight, and add accelerometer assessment among parents. In addition, the definition of NBGM should be further clarified to better understand if parent sleep disturbance occurs from CGM alarms or from actively conducting finger pricks. Tracking sleep patterns and sleep quality in parents would be helpful in more thoroughly assessing HRQOL in families managing T1D. Furthermore, it would be useful to observe longitudinal changes in sleep and HRQOL as children transition onto using CGM. With a better understanding of the relationship, more meaningful conclusions can be drawn about the positive or negative impacts of CGM use on sleep and HRQOL.

\section{Author Disclosure Statement}

No competing financial interests exist.

\section{Funding Information}

This research was supported by grant DP3DK103998-01 from the National Institute of Diabetes and Digestive and Kidney Diseases (last author PI).

\section{References}

1. Nam S, Chesla C, Stotts NA, Kroon L, Janson SL: Barriers to diabetes management: patient and provider factors. Diabetes Res Clin Pract 2011;93:1-9.

2. Meltzer LJ, Moore M: Sleep disruptions in parents of children and adolescents with chronic illnesses: prevalence, causes, and consequences. J Pediatr Psychol 2007;33:279291.

3. Jaser SS, Foster NC, Nelson BA, et al.: Sleep in children with type 1 diabetes and their parents in the T1D exchange. Sleep Med 2017;39:108-115.

4. Reutrakul S, Thakkinstian A, Anothaisintawee T, et al.: Sleep characteristics in type 1 diabetes and associations with glycemic control: systematic review and metaanalysis. Sleep Med 2016;23:26-45.

5. Caruso NC, Radovanovic B, Kennedy JD, et al.: Sleep, executive functioning and behaviour in children and adolescents with type 1 diabetes. Sleep Med 2014;15:1490 1499.
6. Hirshkowitz M, Whiton K, Albert SM, Alessi C, et al.: National Sleep Foundation's updated sleep duration recommendations. Sleep Health 2015;1:233-243.

7. Jaser SS, Lord JH, Simmons JH, Malow BA: Brief report: sleep disturbances in young children with type 1 diabetes. Diabetes Res Clin Pract 2016;120:232-234.

8. Feeley CA, Clougherty M, Siminerio L, et al.: Sleep in caregivers of children with type 1 diabetes. Diabetes Educ 2018;45:80-86.

9. Monaghan MC, Hilliard ME, Cogen FR, Streisand R: Nighttime caregiving behaviors among parents of young children with Type 1 diabetes: associations with illness characteristics and parent functioning. Fam Syst Health 2009;27:28.

10. Monaghan M, Herbert LJ, Cogen FR, Streisand R: Sleep behaviors and parent functioning in young children with type 1 diabetes. Child Health Care 2012;41:246-259.

11. Burckhardt MA, Roberts A, Smith GJ, et al.: The use of continuous glucose monitoring with remote monitoring improves psychosocial measures in parents of children with type 1 diabetes: a randomized crossover trial. Diabetes care 2018;41:2641-2643.

12. Landau Z, Rachmiel M, Pinhas-Hamiel O, et al.: Parental sleep quality and continuous glucose monitoring system use in children with type 1 diabetes. Acta Diabetol 2014;51: 499-503.

13. Barnard K, Crabtree V, Adolfsson P, et al.: Impact of type 1 diabetes technology on family members/significant others of people with diabetes. J Diabetes Sci Technol 2016;10: 824-830.

14. Van Name MA, Hilliard ME, Boyle CT, et al.: Nighttime is the worst time: parental fear of hypoglycemia in young children with type 1 diabetes. Pediatr Diabetes 2018;19: 114-120.

15. Tudor-Locke C, Barreira TV, Schuna Jr JM, et al.: Fully automated waist-worn accelerometer algorithm for detecting children's sleep period time separate from 24-h physical activity or sedentary behaviors. Appl Physiol Nutr Metab 2013;39:53-57.

16. Barreira TV, Schuna JJ, Mire EF, et al.: Identifying children's nocturnal sleep using 24-h waist accelerometry. Med Sci Sports Exerc 2015;47:937-943.

17. Varni JW, Burwinkle TM, Jacobs JR, et al.: The PedsQL ${ }^{\text {TM }}$ in type 1 and type 2 diabetes: reliability and validity of the Pediatric Quality of Life Inventory ${ }^{\mathrm{TM}}$ generic core scales and type 1 diabetes module. Diabetes Care 2003;26:631637.

18. Vandagriff JL, Marrero DG, Ingersoll GM, Fineberg NS: Parents of children with diabetes: what are they worried about? Diabetes Educ 1992;18:299 302.

Address correspondence to: Randi Streisand, PhD

Children's National Hospital Center for Translational Research 6th Floor Main, CTR

111 Michigan Avenue NW Washington, DC 20010

E-mail: rstreis@childrensnational.org 